\title{
A large-area flexible tactile sensor for multi-touch and force detection using electrical impedance tomography
}

This paper was downloaded from TechRxiv (https://www.techrxiv.org).

LICENSE

CC BY 4.0

SUBMISSION DATE / POSTED DATE

$18-12-2021 / 22-12-2021$

\section{CITATION}

Wang, Xiaojie; Chen, Haofeng; Ma, Gang; yang, xuanxuan; geng, jialu (2021): A large-area flexible tactile sensor for multi-touch and force detection using electrical impedance tomography. TechRxiv. Preprint. https://doi.org/10.36227/techrxiv.17263505.v1

$\mathrm{DOI}$

10.36227/techrxiv.17263505.v1 


\title{
A large-area flexible tactile sensor for multi-touch and force detection using electrical impedance tomography
}

\author{
Haofeng Chen, Xuanxuan Yang, Peng Wang, Jialu Geng, Gang Ma, Xiaojie Wang
}

\begin{abstract}
For large area robot skin design, the distribution of rigid components and wires in traditional array sensors leads to the decrease of the flexibility and extensibility of sensors. The flexible sensors based on non-invasive electrical impedance tomography (EIT) can avoid these shortcomings. However, design of a large-area flexible tactile sensors using EIT technique is still challenging due to the tradeoff between manufacturability and spatial resolution. In this study, we proposed a novel non-array EITbased tactile sensor which is made of a porous elastic polymer and ionic liquid. The sensor free from internal array electrodes is straightforward to manufacture and can cover a large area with low cost. To improve the spatial resolution and touch sensitivity, we adopted a deep learning scheme Pyramid Scene Parsing Network (PSPNet) to postprocess the originally reconstructed images to enhance the sensor tactile perception. With this data-driven method, we achieved a singlepoint position detection error of $7.5 \pm 4.5 \mathrm{~mm}$ without using internal electrodes. To overcome the location dependency of EIT sensing problems, we proposed a method of sub-regional fitting to calibrate the distributed forces for the large-area flexible tactile sensor and obtained a quantitative relationship between the touch force and EIT measurement for the entire sensing area for continuous sensing. The real-time performance of the proposed prototype sensor system demonstrated that we can achieve more accuracy of multi-touch and distributed force detection on the non-array sensors for potential applications in human-robot interfaces.
\end{abstract}

Index Terms_Electrical impedance tomography (EIT), force and tactile sensing, deep learning.

\section{INTRODUCTION}

$\mathbf{F}$ LEXIBLE tactile sensors can be seamlessly integrated onto any surface to detect environmental stimuli, either from mechanical or biological systems. They are conventionally fabricated using polymeric materials based on MEMS technology [1]. According to the sensing mechanism, tactile sensors can typically be categorized into capacitive sensors, piezoelectric sensors, piezoresistive sensors, optical sensors, and strain flexible sensors [2], [3].Flexible sensors can be applied in the fields of robotics, medicine, healthcare, and biology [1]-[5].

Tactile sensing, as a human-robot interface (HMR) technology, plays a crucial role in ensuring a safe and intelligent interaction between humans and robots. Robots equipped with flexible tactile sensors provide capabilities to gain the sense

Corresponding author: Gang Ma (e-mail: magang93@ustc.edu.cn) Xiaojie Wang(e-mail: xjwang@iamt.ac.cn)

Haofeng Chen, Xuanxuan Yang Jialu Geng and Xiaojie Wang are with the Institute of Intelligent Machines, Hefei Institutes of Physical Science, Chinese Academy of Sciences, Hefei 230031, China.

Haofeng Chen, Xuanxuan Yang and Gang Ma are with Department of Precision Machinery and Precision Instrumentation, University of Science and Technology of China, Hefei, Anhui 230026, China.(e-mail: magang93@ustc.edu.cn)

Peng Wang are with Shenzhen Apulis Technology Inc., Shenzhen, China of touch by detecting contact positions and forces with their surroundings. This not only assists robots in moving and operating safely in unstructured environments but also makes them safer when working near people. However, to realize tactile functions in robotics, large-area flexible tactile sensors with distributional sensing capability are required.

At present, the most widely used flexible sensors are array sensors consisting of a series of sensing units. However, array sensors are complex, with tiny communication units that make manufacturers complicated and expensive. On the other hand, to transmit data from large-scale sensor arrays, a large number of wires are usually arranged inside the sensor. The distribution of wires not only causes electromagnetic noise but also reduces the flexibility and stretchability, thus limiting their practical applications [4], [5].

Recently, a non-array tactile sensing technology based on electrical impedance tomography (EIT) has emerged and has shown a promising solution for whole-body touch sensing in robots [6], [7]. EIT is a special electrical detection method that is generally used for nondestructive and radiation-free testing. In the field of flexible tactile sensing, this technology constructs an image of the internal conductivity distribution by processing the potential measurement data from the boundary of the soft conductive material. Noninvasive imaging technology could realize a "one-piece" structure of a flexible 
tactile sensor without internal wires and units. The EITbased flexible tactile sensor can obtain the internal conductivity distribution of an electrically conductive body through its boundary electrodes. The position and amplitude of the touching force can be estimated according to the conductivity distribution and intensity. EIT-based flexible tactile sensors have advantages over conventional flexible sensors due to their simple structures, low cost and continuous sensing capacity.

However, building a large-area tactile sensor based on EIT technology is still challenging. Compared with conventional array approaches, EIT-based techniques have shortcomings in their relatively low spatial and temporal resolutions and limited ability to discriminate between pressure intensities and contact areas. All these issues are associated with the size of the sensors, the number of electrodes and the materials used in constructing the sensors. Among them, the characteristics of the sensing materials play a crucial role in their performance. In general, an ideal material for EIT-based flexible tactile sensors should be large-area compliance, facile fabrication and low-cost and have a highly sensitive conductivity in response to external stimuli [6] . Many kinds of soft conductive materials have been explored to develop EIT-based sensors. One of the first attempts to design EIT-based tactile sensors was proposed by Kato et al. [8] . Their prototype sensor system was made of pressure-sensitive conductive rubber. However, the rubber materials were less stretchable and had high hysteresis, which were not suitable for real-time sensing. Yao et al. [9] manufactured conducive fabrics to improve the sensor performance by reducing the material hysteresis behavior. Compared to conductive rubber, the conductive fabric is more stretchable and has less hysteresis. However, the conductivity changes only in the stretching direction, which is not applicable for normal force detection. Tallman et al. [10] fabricated carbon nanofiber/polyurethane nanocomposites as highly flexible conductors to design an EIT-based flexible strain sensor. Lee et al. [11] also prepared multiwall carbon nanotube (MWCNT)-silicone elastomer nanocomposites and implemented them with an anisotropic EIT technique to make multi-point and multi-directional strain mapping sensors. Although soft nanocomposites have demonstrated some advantages in making large-area flexible tactile sensors, it is still challenging to achieve a continuous and homogeneous conductivity distribution over the entire sensing area. Chossat et al. [12] proposed a novel EIT-based tactile sensor using microchannels filled with ionic liquid. This method makes changes in local impedance more repeatable and predictable compared to using conductive rubber. However, the sensor discrete networks implemented with microfluidic channels fail to detect the continuous force over the entire sensing area. Zhao et al. [13] proposed an ionic liquid-based distributed tactile sensor without using microchannels. The sensor was simply made by filling a circular tank with a liquid conductor and sealing the top with an elastic shell. The pressure applied to the elastic top was detected using the EIT imaging method. However, the performance of the tactile sensor was very poor, and it can only achieve a simple touch perception.

Increasing the number of electrodes can improve the performance of the EIT-based tactile sensor, but it also increases the computation time. In our previous study [14] , we proposed an optimal EIT drive pattern based on feature selection and model explanation, which can reduce the number of electrodes and achieve a performance trade-off between the accuracy and time response for real-time EIT tactile sensing. Recently, multielectrodes combined with an adaptive optimal measurement algorithm have been proposed for large-area tactile sensors based on electrical resistance tomography (ERT) [15] . To improve the spatial resolution, internal array electrodes are used in their work. However, numerous internal electrodes result in more internal wires and make the EIT sensing system more complicated.

Additionally, the spatial performance of EIT-based tactile sensors depends heavily on the quality of the reconstructed images. Mathematically, the EIT reconstruction problem is an ill-posed nonlinear inverse problem [16], [17] , and has to be addressed with an appropriate reconstruction algorithm. Traditional iterative imaging algorithms have been used in the field of EIT imaging, such as Tikhonov regularization and Landweber iterative algorithms [18]. However, traditional iterative algorithms adopting linear approximation would cause the loss of important imaging information, resulting in distortion of the reconstructed image. Recently, machine learning has been proposed to address the low spatial resolution problem in EITbased tactile sensors [19]-[22] . For example, Duan et al. [19] fabricated an EIT-based tactile sensor using piezo-resistive fabric and used deep learning as post-image processing to improve image quality and achieved two-point touch detection. Park et al. [20] used a simple deep neural network (DNN) to solve EIT inverse problems and improved the reconstruction accuracy for their tactile sensors. Later, they presented an EITbased tactile sensor with high spatial resolution, sensitivity, localization accuracy, and generalized reconstruction capabilities with the aid of a novel EIT neural network (EIT-NN). The performance of the tactile sensor was very good, but their sensor inevitably adopts an array structure with $20 \times 20$ conductive fabric patches attached to neoprene foam, which increases the complexity of manufacturing [21]. Husain et al. [22] applied a machine learning-driven method for both solving the EIT inverse problem and image postprocessing on an EIT-based tactile sensor. They could recognize fundamental geometric objects such as circles, rectangles, and triangles with their method. However, their method is still in the simulation stage without prototype examination. Compared with linear and nonlinear algorithms, machine learning can obviously achieve better results in solving EIT inverse problems. Its main drawback is its poor prediction ability on unseen data [23] , and it is sensitive to noise and electrode position movement. In contrast, combining a linear solver and postprocessing using machine learning can efficiently avoid these issues. In our previous work [24], we proposed a PSPNet network based on MobileNet for EIT image postprocessing and reduced the size error and position error of reconstruction.

In this paper, we proposed a novel nonarray EIT-based tactile sensor using a porous structure and ionic liquid. The porous material is made from a flexible polyurethane foam, which has excellent elastic properties to ensure that the sensor has a good mechanical response to external stimuli. 
The changes in impedance are directly related to the volume changes of the ionic liquid inside the porous material. To improve the imaging resolution and touch visibility, a deep learning scheme PSPNet as postprocessing is adopted, which can effectively recover the sharp contour of targets and improve the image reconstruction accuracy by completing pixel-level classification. By using this data-driven method, we can improve the spatial resolution of the tactile sensor; thus, the originally indistinguishable touch points were successfully distinguished. Based on the reconstructed image, we established the mapping relationship between the force and the reconstructed conductivity through the subregion fitting method and obtained a good linear relationship between the actual force and the predicted force. We achieved more accurate distributed force detection on nonarray sensors, which provides the potential for the application of tactile sensors to robot tactile perception.

\section{Methods AND SYSTEM DESIGN}

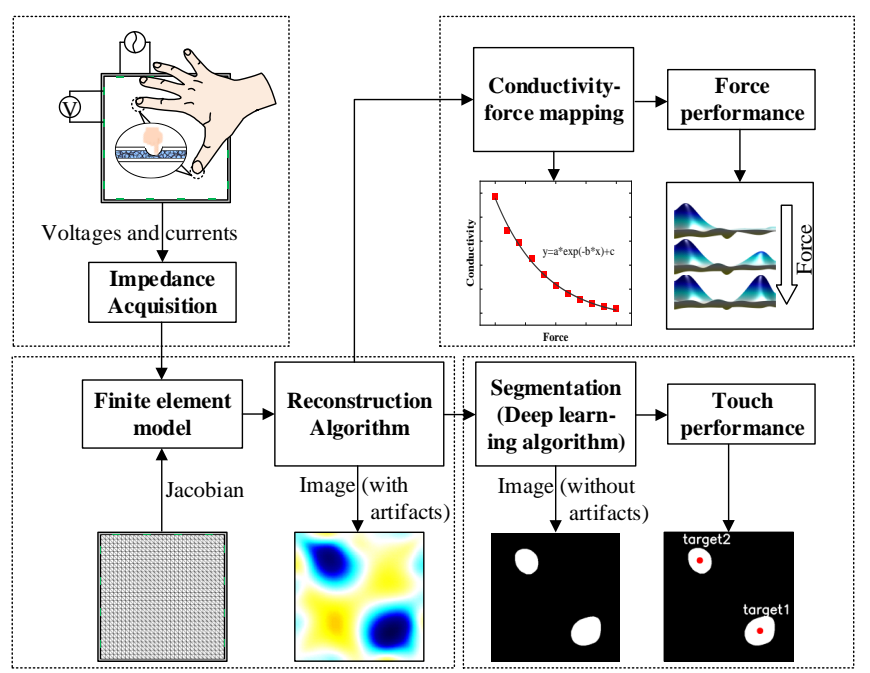

Fig. 1. Overview of the EIT-based tactile sensing system.

Figure 1 shows the overall structure of the proposed tactile sensing system, which consists of four parts: impedance data acquisition, imaging reconstruction utilizing conventional EIT imaging algorithms, image segmentation using a deep learning algorithm, and force perception based on the mapping relationship between the measured conductivity and the applied force. The soft tactile sensor is made of a porous soft material filled with ionic liquids. When a force is applied, the ionic liquid is discharged, and as a result, the conductivity changes. The impedance acquisition system captures the conductivity changes by measuring the voltages and currents using multiple electrodes connected to the boundary of the sensing domain. The finite element model (FEM) and reconstruction algorithm are employed to solve EIT forward and inverse problems to reconstruct the conductivity distribution. The reconstruction images provide two functions. One is to obtain touch information through the deep learning algorithm for image segmentation. Another is to estimate the magnitude of the force by mapping the relationship between the conductivity and the applied force.

In this section, we first illustrate the working principle and fabrication procedure of the EIT-based sensor. Then, we describe a customized design of a data acquisition board. After that, we introduce a reconstruction algorithm for solving the EIT inverse problem. Finally, we propose a deep learning algorithm as postprocessing for image segmentation to improve the imaging resolution.

\section{A. Sensing Principle and Sensor Fabrication}
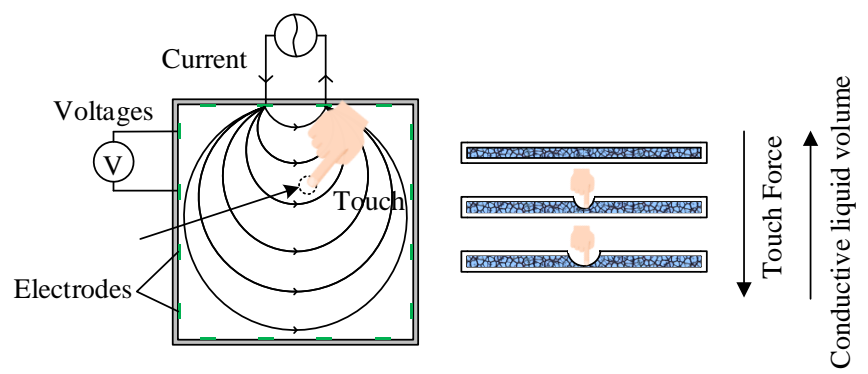

Fig. 2. An illustration of the principle of the flexiable EIT-based tactile sensor using porous structures and ionic liquids. A current stimulation is connected between one pair of electrodes and the voltage is measured from other pairs. The conductive liquid volume of the touch position changes in accordance with the touch force. The local impedance changes in the form of an image are estimated from current stimulations and voltage measurements.

The sensing principle of the flexible EIT-based tactile sensor is illustrated in Figure 2. The conductive liquid filled in a porous elastic polymer provides sensitive impedance changes in response to a touch force. The liquid underneath the contact region presses out when subjected to an applied force. As a result, the local impedance increases almost linearly corresponding to the volume changes of the conductive liquid. For the maximum compression condition, most of the conductive liquid is squeezed out; therefore, the contact area tends to be nonconductive. Unlike many other soft conductive materials, such as piezoresistive rubbers and conductive nanocomposites used for flexible EIT-based tactile sensors, the proposed conductive flexible materials combining elastic porous structures and conductive liquids provide continuous, linear changes in impedance with respect to touch forces.

The manufacturing procedure of the sensing materials for the EIT-based tactile sensor is shown in Figure 3. They are made of a soft polyurethane foam, Ecoflex substrates, and ionic liquids. Following the method in our previous work [25], we prepared soft porous polyurethane foam with a size of 20 $\mathrm{mm} \times 20 \mathrm{~mm} \times 5 \mathrm{~mm}$ and used homemade metal molds to cast the Ecoflex substrate. Tap water containing ionic $\mathrm{Ca}, \mathrm{Mg}, \mathrm{Cl}$, etc. was selected as the ionic liquid to fill the foam, and its conductivity was approximately $125-300 \mu \mathrm{S} / \mathrm{cm}$. Sixteen silver electrodes are evenly placed on the boundary of the sensor, and soft polyurethane foam is placed on the Ecoflex substrate. Then, the ionic liquids are poured into soft polyurethane foam. Finally, the Ecoflex substrates and Ecoflex cover were sealed with silicone sealant. 


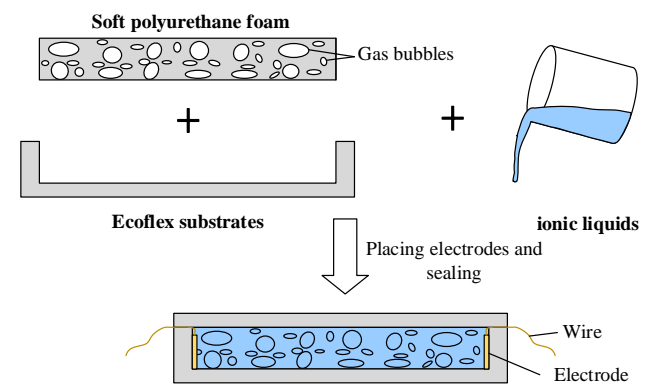

Fig. 3. Fabrication of flexiable EIT-based tactile sensor using porous structures and ionic liquids. A typical soft polyurethane foam is put on the Ecoflex substrates. Then place and fix electrodes evenly on the boundary of the sensor and pour ionic liquids. Finally, seal the Ecoflex substrates and Ecoflex cover with a silicone sealant.

\section{B. Impedance data acquisition board}
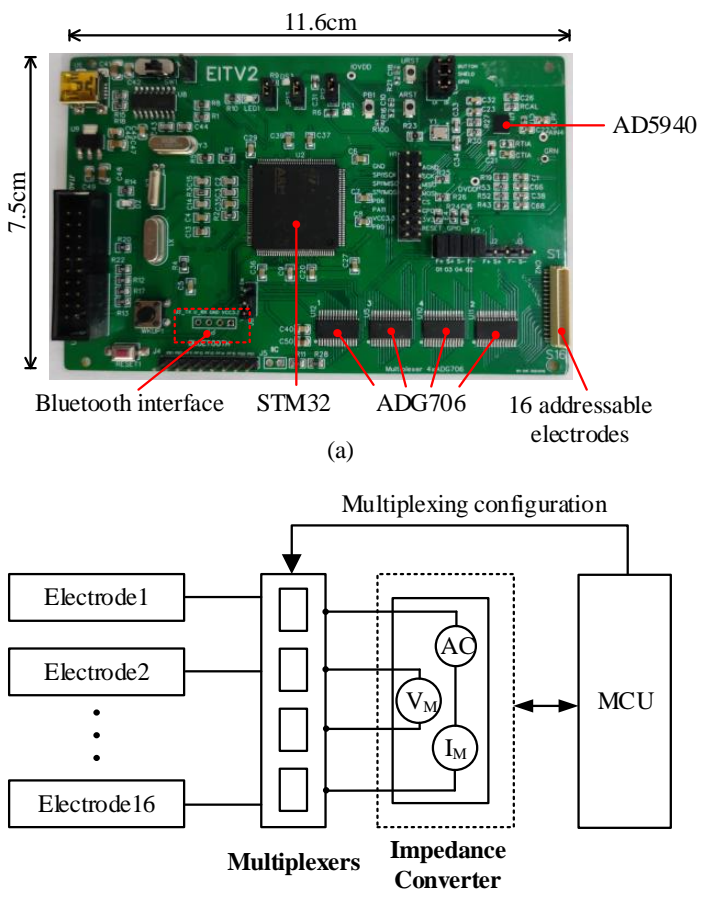

(b)

Fig. 4. Impedance data acquisition system. (a) The data acquisition board. (b) A circuit schematic of our data acquisition board.

We designed a customized impedance data acquisition board (see Figure 4 (a)). The corresponding circuit schematic of the proposed data acquisition board is shown in Figure 4 (b), which comprises 16 electrodes, 4 analog multiplexers, an impedance converter, and a microcontroller unit (MCU). The 16 electrodes are evenly placed at the boundary of the sensing domain for current injection and voltage measurements. Two 16-to-1 multiplexers (ADG706 chip) connect the current injection terminal and current measurement terminal to any two electrodes, making up the current-injection pair. The voltage measurement terminals are connected to two more multiplexers, forming the voltage-measurement pair. To simplify our system and increase its speed, we employed a two-terminal scheme for data acquisition, in which the current injection and voltage measurement were carried out at the same electrode pair [26]. An AD5940 analog chip, which is a high precision, ultralow power, analog front-end system, was selected as an impedance converter for impedance measurement [27]. As shown in Figure 4(b), this chip is composed of a precision AC voltage source, a high precision current meter (IM) and a precision differential voltage meter (VM). A constant excitation signal of $40 \mathrm{kHz}$ is adopted in the AC voltage source, which has been used in many EIT systems [14], [28], [29]. Additionally, the digital filtering method is applied to improve the signal-to-noise performance and measurement accuracy.

A Cortex M3 MCU operating at $72 \mathrm{MHz}$ (STM32F103ZET6 [30]) is applied to control the multiplexer to connect the impedance converter chip AD5940 and 16 addressable electrodes and process the impedance data from the AD5940. In addition, a Bluetooth interface for wireless data transmission is also considered in the design. The impedance data between all electrode pairs were measured sequentially without repetition, which resulted in 120 independent values (with sixteen electrodes). The system achieved an output data rate (ODR) of $200 \mathrm{~Hz}$ (approximately 1.6 frames per second). Then, the impedance data obtained by the impedance converter are transmitted to a computer by a microcontroller (MCU) via universal asynchronous receiver transmitter (Uart) communication for further processing and image reconstruction.

\section{Image Reconstruction and Segmentation}

The essence of image reconstruction for EIT is to solve the inverse problem, which is an ill-conditioned nonlinear problem. Due to a considerable amount of noise in the EIT measurement system, image reconstruction involves a more complicated calculation process, and the spatial resolutions of the resulting images are quite low.

In this study, we first employed a traditional imaging algorithm called Newton's one-step error reconstructor (NOSER) for image reconstruction [31]. This algorithm is less computationally expensive and can achieve fast and robust operations for real-time imaging. However, this method is unable to clearly reflect the characteristics of the object owing to the noise and the accuracy limitation of the measurement system. To address this problem, we proposed a deep learning scheme called the pyramid scene parsing network (PSPNet) [32] based on MobileNet [33] to postprocess the results of reconstructed images to improve the performance of tactile perception.

The architecture of the algorithm is illustrated in Figure 5. For a given input image, the pretrained MobileNet is used to extract the feature map of the reconstructed image. We only used the last output of MobileNet as the next input. PSPNet adopted linear interpolation in resizing images instead of the upsampling trick. With the expansion of the image size (h: height, w:width), the final feature map size is $1 / 8$ of the input image. The core focus is to use an asynchronous long stride and an average pooling layer of different sizes for pooling and then resize the pooling results. After the image height and width were uploaded, they were concatenated. PSPNet used global average pooling and feature fusion. The features 
are in a pyramid structure, which can introduce more context information into the semantic segmentation algorithm, which can reduce many missegmentations. When we adapted PSPNet to EIT imaging, it can effectively remove image artifacts and reconstruct a clearer image.

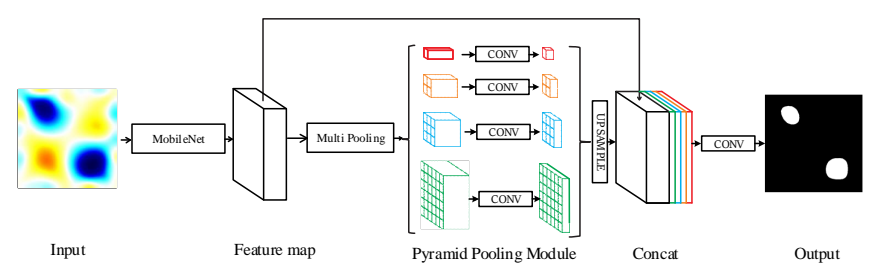

Fig. 5. Overview of our proposed PSPNet [24].

For training and testing, the PyTorch framework [34] is used to build the deep learning model. The experimental dataset consists of a total of 780 pairs of multipoint touch (from one-point touch to four-point touch) reconstructed images, including $90 \%$ of the training set and $10 \%$ of the validation set. of the validation set. The size of the image input to the network is $612 \times 612$. We employed batch normalization to all hidden layers for robust convergence during training. The batch size was empirically set to 24 , and the class number was 2. The optimizer ADAM with a learning rate of 0.001 is used for training with 100 epochs. For the model test, obviously different test data are employed.

\section{EXPERIMENTS}

The experimental apparatus for evaluating the tactile sensor performance is shown in Figure 6. All the tests are conducted on a universal testing machine (E44.104, MTS, US) comprising an integrated, digital closed-loop linear actuator. It is equipped with a $100 \mathrm{~N}$ load cell. A $200 \mathrm{~mm} \times 200 \mathrm{~mm}$ positioning plate with $19 \times 19$ holes is connected to the MTS load cell through a metal clamp and used to fix a cylindrical indenter with a diameter of $15 \mathrm{~mm}$. The indenters are used to apply touch force on the tactile sensor. The touch positions can be recognized by the hole positions where the indenters are installed.

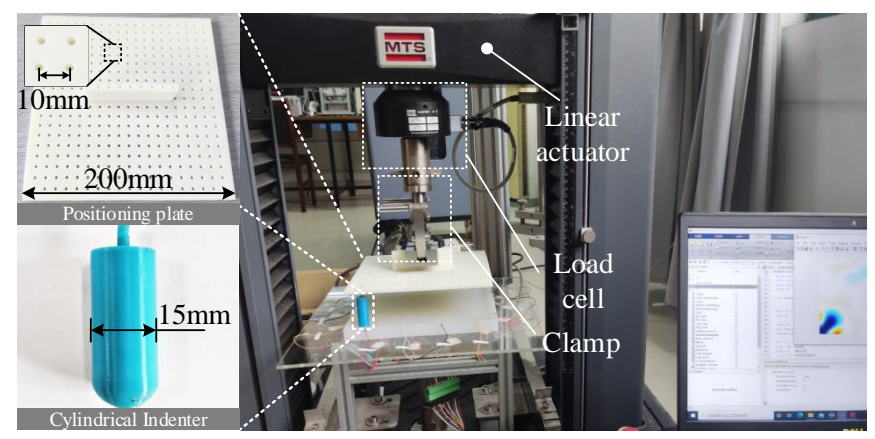

Fig. 6. Experimental apparatus for evaluating the tactile sensor performance.

\section{A. Single-Touch Indentation Test}

A single-touch indentation test was carried out to assess the touch position error (PE) of the proposed EIT-based sensor.
A constant pressing force of $1.2 \mathrm{~N}$ was applied to a set of points formed by a $5 \times 5$ array (as shown in Figure 8) through an indenter $15 \mathrm{~mm}$ in diameter. The interval of two adjacent points is $40 \mathrm{~mm}$. The testing area of the sensor leaves from its border of $20 \mathrm{~mm}$. In every indentation test, the estimated location by the EIT-based sensor was compared with the real touch position where the indenter was located to obtain the PE value.

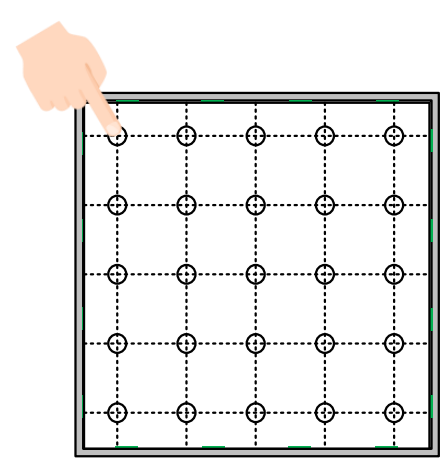

Fig. 7. Schematic diagram of single touch test.

The estimated positions can be obtained by calculating the centroid of the reconstructed image $\left(x_{c}, y_{c}\right)$ in two steps. First, the traditional imaging algorithm NOSER is performed to reconstruct the images (Figure 8 (a)). Then, the deep learning scheme PSPNet based on MobileNet is used to obtain the postprocessing images (Figure 8 (b)). From the postprocessing image, the centroid of touch locations can be calculated as follows.

$$
\left(x_{c}, y_{c}\right)=\left(\frac{\sum[P]_{i} x_{i}}{\sum[P]_{i}}, \frac{\sum[P]_{i} y_{i}}{\sum[P]_{i}}\right)
$$

where $[P]_{i}$ is the pixel value of the post-processing image at $\left(x_{i}, y_{i}\right)$.

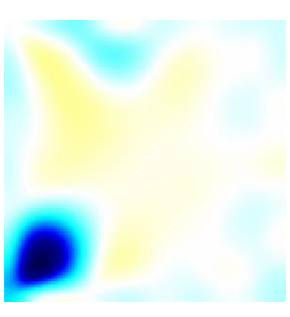

(a)

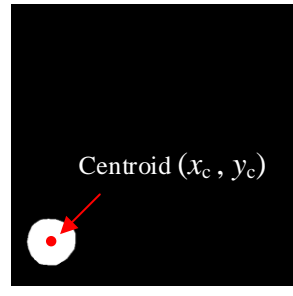

(b)
Fig. 8. The contact locations were estimated by two steps. (a) Step 1: Obtain the reconstructed image by a traditional imaging algorithm. (b) Step 2: Calculate the centroids of the postprocessing image obtained by a deep learning algorithm.

For each indentation test, up to 30 time series samples were recorded, and a total of 750 samples were used to estimate the 25 locations. Figure 9 presents the error vectors between actual touch positions and the estimated locations. For the same size tactile sensor, the proposed soft tactile sensor based on porous structures and ionic liquids achieved a single-point position detection error of $7.5 \pm 4.5 \mathrm{~mm}$ without using internal electrodes, whereas [21] used an internal electrode with a more 
complicated array structural design and only achieved a position detection rate error of $8.2 \pm 4.3 \mathrm{~mm}$. This demonstrated that the proposed deep learning PSPNet algorithm can improve the spatial performance of the tactile sensor very well.

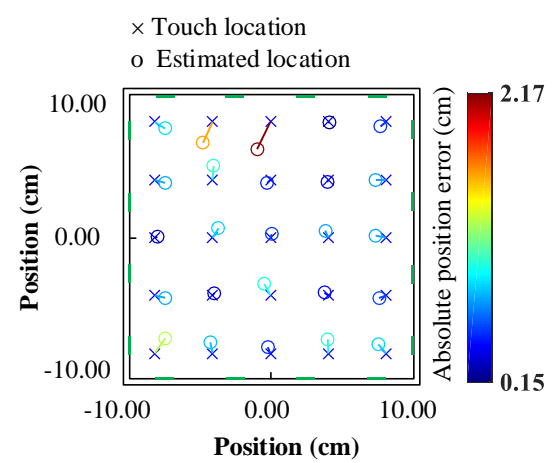

Fig. 9. Touch position error results. The cross mark represents the actual touch location, whereas the circle represents the average of the estimated location. The error vector is also drawn for each contact location.

\section{B. Multi-Touch Indentation Test}

To further evaluate the spatial resolution of the proposed large-area tactile sensor, a series of multitouch indentation tests was performed. In addition to the two-point discrimination test [21], which has been presented to examine a deep neural network-based EIT method for large-area tactile sensing, we proposed a comprehensive testing protocol including two-point, three-point and four-point discrimination tests for evaluating the tactile spatial acuity of our sensor. Since the sensitivity to intensity of the EIT-based sensor varies across the sensing area, the spatial performance of the sensor is location-dependent [35]. Therefore, we selected two typical cases for the two-point discrimination tests similar to reference [21]. As shown in Figure 10 (a), Case 1 represents the high sensitivity region (close to the electrodes); case 2 represents the low sensitivity region (close to the center of sensor). The arrangements of the test locations for the three-point and four-point discrimination tests are shown in Figure 10 (b) and Figure 10 (c), respectively. In both three-point and four discrimination tests, we fixed the positions of two points with a distance of $10 \mathrm{~cm}$, and the center of the two points was 8 $\mathrm{cm}$ from the center of the sensor. The third and fourth points vary according to the distances of $h$ and $d$, as shown in Figure 10 (b) and (c).

To perform the multipoint discrimination tests, corresponding numbers of identical indenters with a diameter of 15 $\mathrm{mm}$ were installed on the positioning plate to apply touch force on the tactile sensor simultaneously. The applied force on each point was approximately $1.2 \mathrm{~N}$. For the two-point discrimination test, the distance between the contact points was incremented at an interval of $2 \mathrm{~cm}$ for case 1 and an interval of $2.8 \mathrm{~cm}$ for case 2 . For the three-point discrimination test, the positions of two cylindrical indenters are fixed $\left(\mathrm{d}_{1}=10 \mathrm{~cm}\right.$, $\mathrm{h}_{1}=8 \mathrm{~cm}$ ), and the third cylindrical indenter is below the two cylindrical indenters with distance from the center $\mathrm{O}$ (written as h) incremented at an interval of $1 \mathrm{~cm}$. Similarly, for the

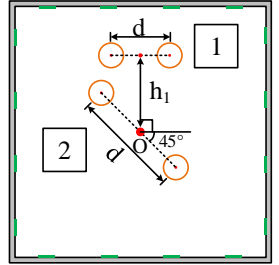

(a)

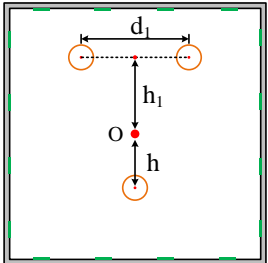

(b)

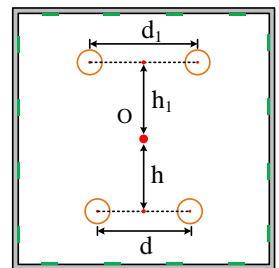

(c)
Fig. 10. Descriptions about the multi-point discrimination test. (a) Locations for the two-point discrimination test. In each case, the twopoint touch stimulus was imposed according to the varying two-point distance (written as d), 1: the line of two points is parallel to the boundary $\left(\mathrm{h}_{1}=8 \mathrm{~cm}\right)$. 2: two points are located on the diagonal. (b) Locations for the three-point discrimination test , the positions of two upper points are fixed $\left(d_{1}=10 \mathrm{~cm}, h_{1}=8 \mathrm{~cm}\right)$, and the third point is below the two points with varing distance from the center $\mathrm{O}$ (written as $\mathrm{h}$ ). (c) Locations for the four-point discrimination test. The positions of two upper points are fixed $\left(d_{1}=10 \mathrm{~cm}, h_{1}=8 \mathrm{~cm}\right)$, and the positions of two lower points are varying in distances $d$ and $h$.

TABLE I

TWO-PoINT Discrimination TESt REsUlts

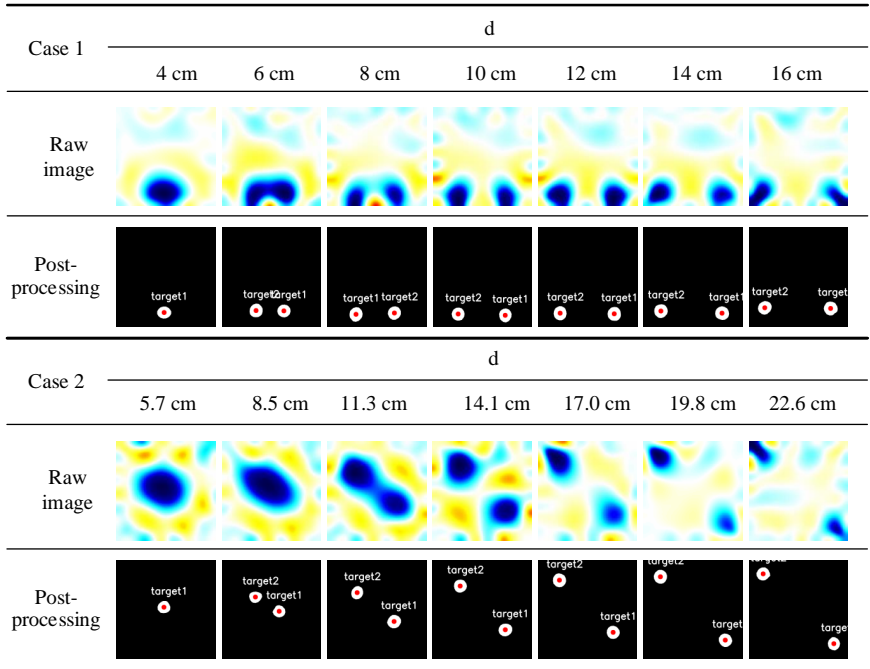

four-point discrimination test, the positions of two cylindrical indenters are fixed $\left(\mathrm{d}_{1}=10 \mathrm{~cm}, \mathrm{~h}_{1}=8 \mathrm{~cm}\right)$. The positions of the two lower cylindrical indenters increase at an interval of $2 \mathrm{~cm}$ in the horizontal direction (written as d, as shown in Figure 10 (c)) and at an interval of $1 \mathrm{~cm}$ in the vertical direction (written as $\mathrm{h}$ as shown in Figure 10 (c)).

TABLE I through TABLE III visualize the comparison results of the originally reconstructed images and postprocessed images. TABLE I shows the results of two-point discrimination tests for cases 1 and 2 . In case 1 , it can be seen that the postprocessing makes the two points $6 \mathrm{~cm}$ apart completely distinguishable in contrast to the raw images reconstructed by the traditional algorithm. In case 2, the two points $8.5 \mathrm{~cm}$ apart can be distinguished by postprocessing, while the traditional algorithm fails to discern them.

TABLE II and TABLE III illustrate the results of the three-point and four-point discrimination tests, respectively. As shown in TABLE II, when the third touch position moves toward the center, the originally reconstructed images become 
TABLE II

THREE-Point Discrimination TESt REsults

\begin{tabular}{|c|c|c|c|c|c|c|c|}
\hline & & & & $\mathrm{h}$ & & & \\
\hline & $1 \mathrm{~cm}$ & $2 \mathrm{~cm}$ & $3 \mathrm{~cm}$ & $4 \mathrm{~cm}$ & $5 \mathrm{~cm}$ & $6 \mathrm{~cm}$ & $7 \mathrm{~cm}$ \\
\hline $\begin{array}{c}\text { Raw } \\
\text { image }\end{array}$ & & & 8 & 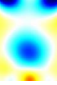 & $\theta$ & 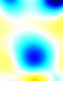 & \\
\hline $\begin{array}{c}\text { Post- } \\
\text { processing }\end{array}$ & $0^{0}$ & 0 & $\begin{array}{ll}0 & 0 \\
\text { torgeti } \\
\text { togt }\end{array}$ & $\begin{array}{c}0 \\
0 \\
\text { torget1 }\end{array}$ & targett & $\begin{array}{c}0 \\
0 \\
\text { targett }\end{array}$ & 0 \\
\hline
\end{tabular}

TABLE III

FouR-POINT DISCRIMINATION TEST REsULTS

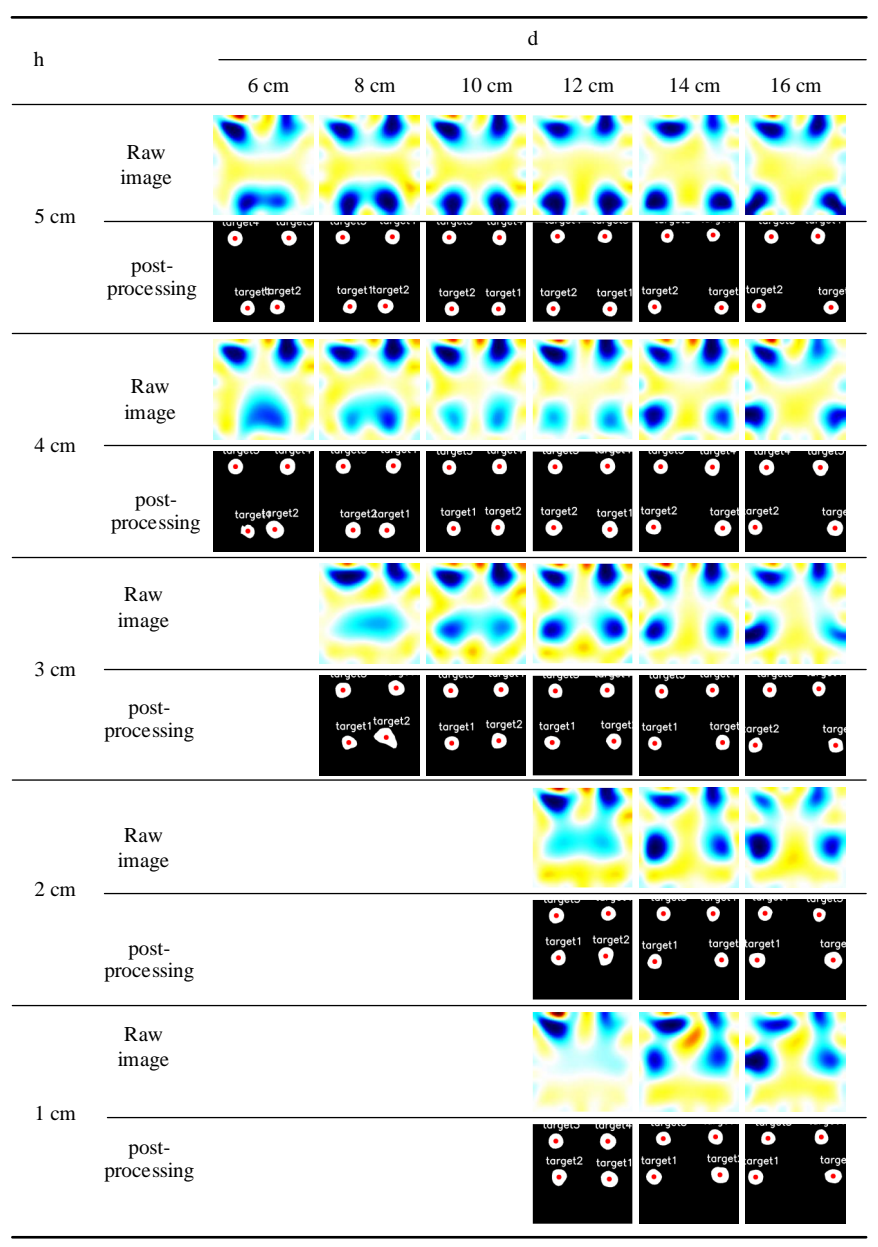

blurry. However, the postprocessing images can effectively recognize all three points even with the third point $1 \mathrm{~cm}$ closer to the center. This clearly demonstrates that the lower sensitivity of the central area for the proposed large-area tactile sensor is greatly improved by using the deep learning scheme to postprocess the raw images.

More complex touch results for the four-point discrimination test associated with 30 different conditions are shown in TABLE III. We found that as the touch point is closer to the center of the sensor, the distinction becomes more difficult. However, our proposed tactile sensor can still achieve good four-point touch discrimination ability. Postprocessing plays a crucial role in improving the spatial resolution of the largearea EIT-based tactile sensor.

Some typical results of the real-time demonstrations of multipoint discrimination tests are shown in Figure 11 (details can be seen in video I). Additionally, by placing six points in an appropriate position, the sensor is able to discriminate up to 6 points in a $20 \mathrm{~cm} \times 20 \mathrm{~cm}$ tactile area within a $6 \mathrm{~cm}$ distance (see video I).
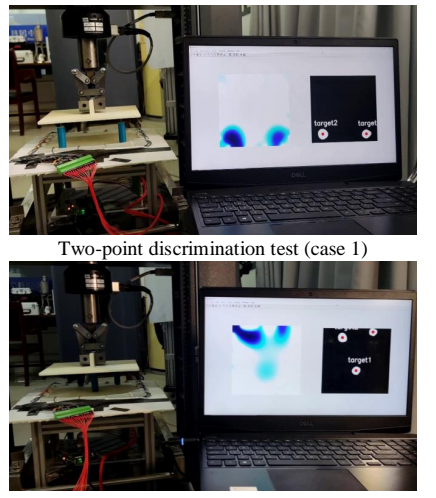

Three-point discrimination test
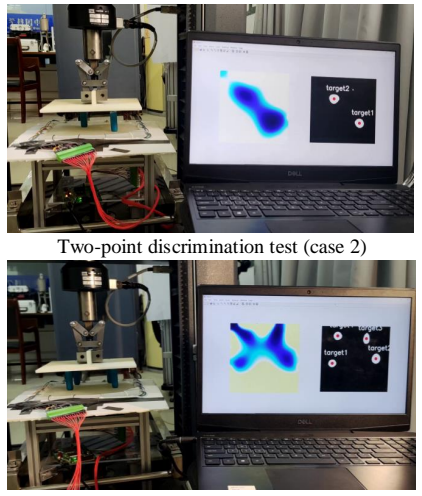

Four-point discrimination test
Fig. 11. Real-time multi-point discrimination demonstration.

\section{Touch Force Calibration}

The reconstructed images allow us to establish the mapping relationship between the strength of a touch force and the image intensity over the entire sensing area. However, the inherent location dependency of tactile sensitivity severely affects interpreting the strength information of a touch force from different locations of EIT-based sensors [35].

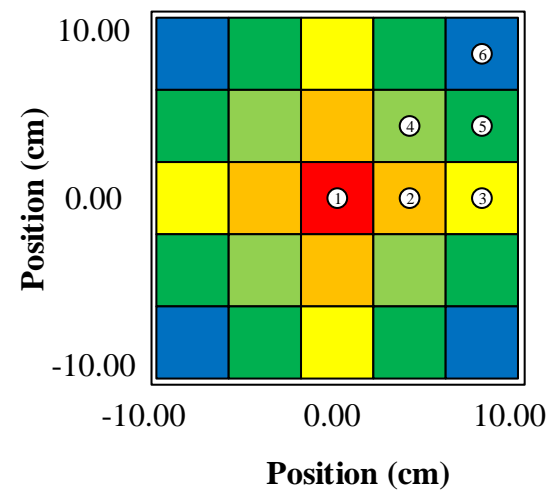

Fig. 12. Schematic diagram of different sensing divisions indicated by the numbers 1-6. The symmetrical areas are marked with the same color representing the same division.

To overcome the location dependency of EIT sensing problems, we proposed a method of subregional fitting to calibrate the distributed forces for our large-area flexible tactile sensor. As shown in Figure 12, we took advantage of the symmetry of the sensor and divided the sensing area into 6 regions marked with different colors and assumed that the tactile sensitivity in each area was constant.

In the calibration tests, a series of touch forces from 0.0 to $2.0 \mathrm{~N}$ were applied at an interval of $0.2 \mathrm{~N}$ on each division 


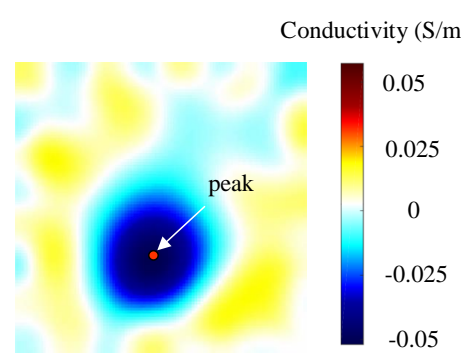

(a)

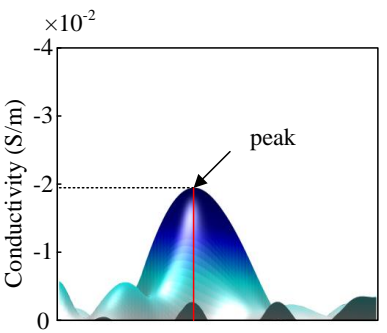

(b)

Fig. 13. The reconstructed image reflecting the distribution of conductivity change under a touch force(left) and its three-dimensional form (right).

marked with numbers 1-6, as shown in Figure 12. Up to 15 time series samples were recorded, and the data were used to reconstruct images for one touch test. Since the amplitude of the applied force directly relates to the peak value of the reconstructed conductivity, we extracted the maximum value of the reconstructed image (as shown in Figure. 13) to calibrate the force sensing capability of the proposed EIT-based tactile sensor.

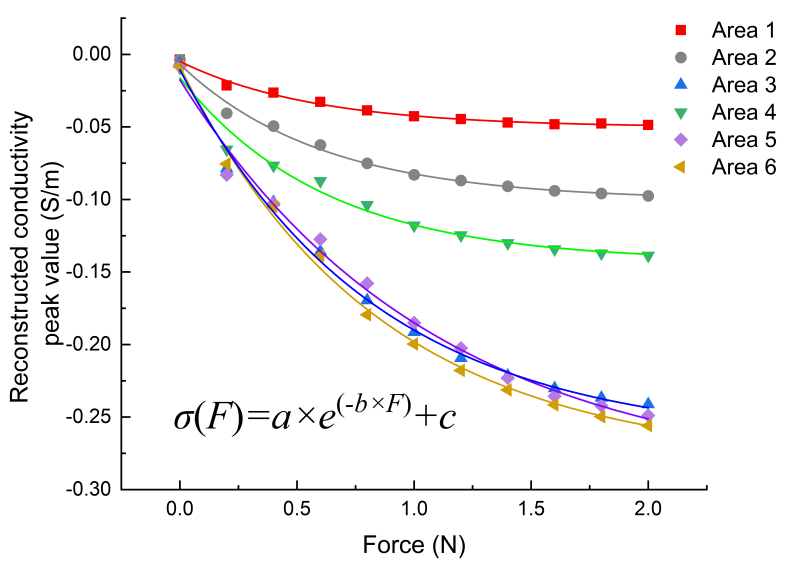

Fig. 14. The plots of calibration results for the touch forces as a function of the peak value of the reconstructed images in different sensing areas. The dots represent the experimental data; the solid lines are fitted curves by an exponential function.

Figure 14 shows the calibration results for the relationships of the touch force and the average reconstructed conductivity peak value in the six areas. Note that the experimental data in each area are fitted very well with an exponential function:

$$
\sigma(F)=a \times e^{(-b \times F)}+c
$$

where $F$ is the touch force, $\sigma$ is the peak value of reconstructed conductivity and $a, b$ and $c$ are coefficients. The best-fit values of $a, b, c$ and corresponding $\mathrm{R}$ squared values for the exponential functions in six different areas are presented in Table IV.

Rewriting (2), we obtain an explicit force function in terms of the peak value of the reconstructed conductivity as follows.
TABLE IV

BEST FITTING PARAMETERS AND R-SQUARED VALUES

\begin{tabular}{ccccc}
\hline Area & $\mathbf{a}$ & $\mathbf{b}$ & $\mathbf{c}$ & $R^{2}$ \\
\hline 1 & 0.045 & 1.745 & -0.050 & 0.992 \\
\hline 2 & 0.094 & 1.639 & -0.100 & 0.990 \\
\hline 3 & 0.256 & 1.209 & -0.266 & 0.995 \\
\hline 4 & 0.127 & 1.608 & -0.143 & 0.979 \\
\hline 5 & 0.277 & 0.926 & -0.295 & 0.991 \\
\hline 6 & 0.276 & 1.133 & -0.287 & 0.997 \\
\hline
\end{tabular}

$$
F=-\frac{1}{b} \ln \frac{\sigma-c}{a}
$$

Therefore, (3) provides a quantitative relationship between the touch force and EIT measurement for the entire sensing area for continuous sensing.

It is interesting to see that the fitting curves of area 3 and area 5 are indistinguishable from that of area 6 , which is located at four corners of the square-size sensor. This unusual phenomenon might be attributed to the nonuniformity of the sensing material.

To validate the force function of (3), we randomly selected touch locations including the six marked areas used for calibration and an applied force in the range of 0.2-1.8 Over 150 samples were recorded in the tests. The results of the real forces and the forces estimated by (3) are shown in Figure 15. Note that there is a good linear relationship between the predicted force and the applied force with a linear correlation coefficient of 0.96 . This result warranted that the proposed method of subregional fitting to calibrate the distributed forces of the tactile sensor was effective. By calculating the rootmean-squared error (RMSE) of the real force and the predicted force, we found that the force performance of the proposed EIT-based tactile sensor has an error of $0.19 \mathrm{~N}$ (10\% of the maximum force). In most cases of human-robot interactions, the accuracy of the tactile sensors is sufficient for applications in discrimination of the applied forces.

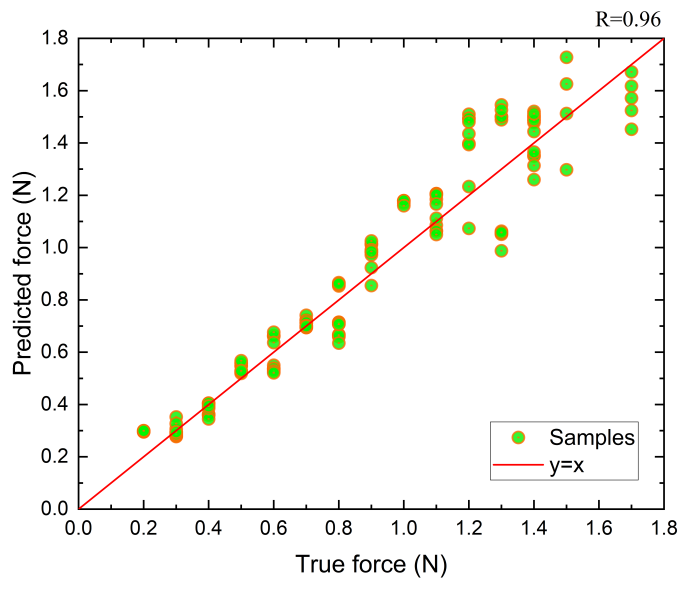

Fig. 15. Force estimation based on subregional fitting method. 
Figure 16 shows some examples of real-time touch tests using the proposed large-area tactile sensor (S video II). Without applying force, the laptop displays a blank picture on the screen. When a touch force by a fingertip is applied on the tactile sensor, the screen will show the touch position and the amplitude of the touch force by a 3-D reconstructed image on the blank picture instantly. At the same time, the touch position and force information is displayed on the screen in red text. When we slowly increase the contact force, the height of the reconstructed image will increase. Each real-time test contains three pieces of information: the touch area (Touch block), the center position of the touch point (Cent), and the force (Force). Combined with visualized three-dimensional conductivity imaging information, we can achieve effective force detection. This demonstrated that our large-area flexible tactile sensor can realize real-time and more accurate distributed force detection, which could provide potential applications for human-robot tactile perception.

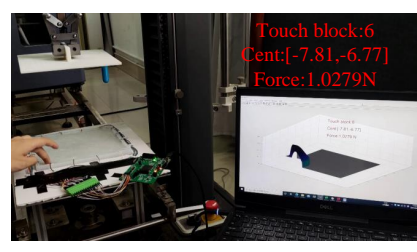

Block 1

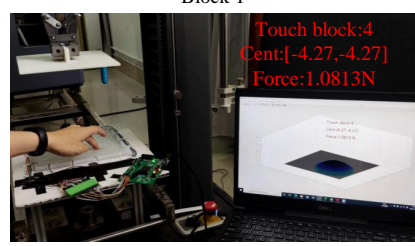

Block 3

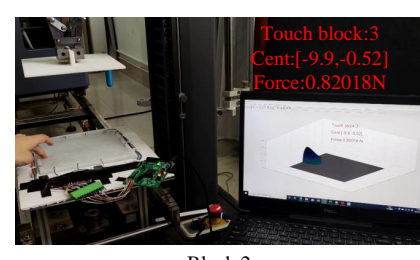

Block 2

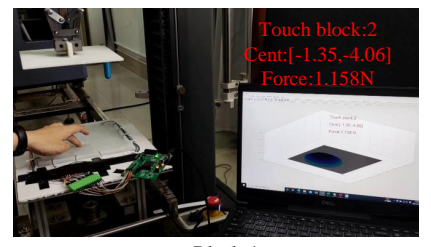

Block 4
Fig. 16. Real-time position and force detection demonstration.

\section{CONCLUSION}

In this study, we developed a large-area flexible tactile sensor for multi-touch and force detection based on EIT technology. A novel design of a sensor material made of a porous elastic polymer and ionic liquid was proposed. The proposed conductive flexible materials combining elastic porous structures and conductive liquids provide continuous, linear changes in impedance with respect to touch forces. A deep learning scheme PSPNet based on MobileNet was adopted to postprocess the originally reconstructed images to improve the performance of tactile perception. By using this data-driven method, we can improve the spatial resolution of the tactile sensor to achieve a single-point position detection error of $7.5 \pm 4.5 \mathrm{~mm}$ without using internal electrodes. To overcome the location dependency of EIT sensing problems, we proposed a method of subregional fitting to calibrate the distributed forces for our large-area flexible tactile sensor and achieved a quantitative relationship between the touch force and EIT measurement for the entire sensing area for continuous sensing. We achieved more accurate distributed force detection on nonarray sensors. The force performance of the proposed EIT-based tactile sensor has an error of 0.19
$\mathrm{N}$ (10\% of the maximum force). Moreover, with this strategy, we are able to achieve multipoint discrimination up to 6 points in a $20 \mathrm{~cm} \times 20 \mathrm{~cm}$ tactile area within a $6 \mathrm{~cm}$ distance.

\section{REFERENCES}

[1] S. Han, H. Peng, Q. Sun, S. Venkatesh, K. Chung, S. C. Lau, Y. Zhou, and V. Roy, "An overview of the development of flexible sensors," Advanced materials, vol. 29, no. 33, p. 1700375, 2017.

[2] S. Chen, K. Jiang, Z. Lou, D. Chen, and G. Shen, "Recent developments in graphene-based tactile sensors and e-skins," Advanced Materials Technologies, vol. 3, no. 2, p. 1700248, 2018.

[3] Y. Al-Handarish, O. M. Omisore, T. Igbe, S. Han, H. Li, W. Du, J. Zhang, and L. Wang, "A survey of tactile-sensing systems and their applications in biomedical engineering," Advances in Materials Science and Engineering, vol. 2020, no. 4047937, 2020.

[4] S. Khan, L. Lorenzelli, and R. S. Dahiya, "Technologies for printing sensors and electronics over large flexible substrates: a review," IEEE Sensors Journal, vol. 15, no. 6, pp. 3164-3185, 2014.

[5] G. Ge, Y. Cai, Q. Dong, Y. Zhang, J. Shao, W. Huang, and X. Dong, "A flexible pressure sensor based on rgo/polyaniline wrapped sponge with tunable sensitivity for human motion detection," Nanoscale, vol. 10, no. 21, pp. 10033-10040, 2018.

[6] D. Silvera-Tawil, D. Rye, M. Soleimani, and M. Velonaki, "Electrical impedance tomography for artificial sensitive robotic skin:a review," IEEE Sensors Journal, vol. 15, no. 4, pp. 2001-2016, 2015.

[7] L. V. Duong and V. A. Ho, "Large-scale vision-based tactile sensing for robot links: Design, modeling, and evaluation," IEEE Transactions on Robotics, vol. 37, no. 2, pp. 390-403, 2021.

[8] Y. Kato, T. Mukai, T. Hayakawa, and T. Shibata, "Tactile sensor without wire and sensing element in the tactile region based on eit method," SENSORS, 2007 IEEE, pp. 792-795, 2007.

[9] A. Yao, C. L. Yang, J. K. Seo, and M. Soleimani, "Eit-based fabric pressure sensing," Computational and mathematical methods in medicine, vol. 2013, no. 405325, 2013.

[10] T. Tallman, S. Gungor, G. Koo, and C. Bakis, "On the inverse determination of displacements, strains, and stresses in a carbon nanofiber/polyurethane nanocomposite from conductivity data obtained via electrical impedance tomography," Journal of Intelligent Material Systems and Structures, vol. 28, no. 18, pp. 2617-2629, 2017.

[11] H. Lee, D. Kwon, H. Cho, I. Park, and J. Kim, "Soft nanocomposite based multi-point, multi-directional strain mapping sensor using anisotropic electrical impedance tomography," Scientific Reports, vol. 7, no. 1, p. 39837, 2017.

[12] J.-B. Chossat, H.-S. Shin, Y.-L. Park, and V. Duchaine, "Soft tactile skin using an embedded ionic liquid and tomographic imaging," Journal of mechanisms and robotics, vol. 7, no. 2, pp. 1-9, 2015.

[13] T. Zhao, C. Wu, and M. Soleimani, "Ionic liquid based distributed touch sensor using electrical impedance tomography," IOP SciNotes, vol. 1, no. 2, p. $025005,2020$.

[14] G. Ma, Z. Hao, X. Wu, and X. Wang, "An optimal electrical impedance tomography drive pattern for human-computer interaction applications," IEEE Transactions on Biomedical Circuits and Systems, vol. 14, no. 3, pp. 402-411, 2020.

[15] K. Park, H. Lee, K. J. Kuchenbecker, and J. Kim, "Adaptive optimal measurement algorithm for ert-based large-area tactile sensors," IEEE/ASME Transactions on Mechatronics, pp. 1-1, 2021.

[16] Z. Wei, D. Liu, and X. Chen, "Dominant-current deep learning scheme for electrical impedance tomography," IEEE Transactions on Biomedical Engineering, vol. 66, no. 9, pp. 2546-2555, 2019.

[17] A. Adler, J. Arnold, R. Bayford, A. Borsic, B. Brown, P. Dixon, T. Faes, I. Frerichs, H. Gagnon, and Y. Garber, "Greit: a unified approach to $2 \mathrm{~d}$ linear eit reconstruction of lung images," Physiological Measurement, vol. 30, no. 6, pp. S35-55, 2009.

[18] Z. Liu, G. Yang, N. He, and X. Tan, "Landweber iterative algorithm based on regularization in electromagnetic tomography for multiphase flow measurement," Flow Measurement and Instrumentation, vol. 27, pp. 53-58, 2012.

[19] X. Duan, S. Taurand, and M. Soleimani, "Artificial skin through supersensing method and electrical impedance data from conductive fabric with aid of deep learning," Scientific reports, vol. 9, no. 1, pp. 1-11, 2019.

[20] H. Park, H. Lee, K. Park, S. Mo, and J. Kim, "Deep neural network approach in electrical impedance tomography-based real-time soft tactile sensor," in 2019 IEEE/RSJ International Conference on Intelligent Robots and Systems (IROS). IEEE, 2019, pp. 7447-7452. 
[21] H. Park, K. Park, S. Mo, and J. Kim, "Deep neural network based electrical impedance tomographic sensing methodology for large-area robotic tactile sensing," IEEE Transactions on Robotics, vol. 37, no. 5, pp. 1-14, 2021.

[22] Z. Husain, N. A. Madjid, and P. Liatsis, "Tactile sensing using machine learning-driven electrical impedance tomography," IEEE Sensors Journal, vol. 21, pp. 11628-11642, 2021.

[23] S. Martin and C. T. M. Choi, "A post-processing method for threedimensional electrical impedance tomography," Scientific Reports, vol. 7, no. 1, p. 7212, 2017.

[24] P. Wang, H. Chen, G. Ma, R. Li, and X. Wang, "Deep learning scheme pspnet for electrical impedance tomography," in Sensors and Smart Structures Technologies for Civil, Mechanical, and Aerospace Systems 2021, vol. 11591. International Society for Optics and Photonics, 2021, p. 1159112.

[25] J. Geng, C. Wang, H. Zhu, and X. Wang, "Effect of the carbonyl iron particles on acoustic absorption properties of magnetic polyurethane foam," in Behavior and Mechanics of Multifunctional Materials and Composites XII, vol. 10596. International Society for Optics and Photonics, 2018, p. 105961W.

[26] J. Chen, L. Xu, Z. Cao, and H. Zhou, "Four-terminal imaging using a two-terminal electrical impedance tomography system," IEEE Transactions on Instrumentation and Measurement, vol. 63, no. 2, pp. 432-440, 2013.

[27] Analog Devices, High Precision, Impedance, and Electrochemical Front End AD5940/5941, Data Sheet. [Online]. Available: https://www.analog.com/media/en/technical-documentation/datasheets/AD5940-5941.pdf.

[28] Y. Zhang and C. Harrison, "Tomo: Wearable, low-cost electrical impedance tomography for hand gesture recognition," in Proceedings of the 28th Annual ACM Symposium on User Interface Software \& Technology. ACM, 2015, pp. 167-173.

[29] Y. Zhang, R. Xiao, and C. Harrison, "Advancing hand gesture recognition with high resolution electrical impedance tomography," in Proceedings of the 29th Annual Symposium on User Interface Software and Technology. ACM, 2016, pp. 843-850.

[30] ST Microelectronics Corporation, STM32F103XE, Datasheet. [Online]. Available: https://www.st.com/resource/en/datasheet/stm32f103ze.pdf.

[31] M. Cheney, D. Isaacson, J. C. Newell, S. Simske, and J. Goble, "Noser: An algorithm for solving the inverse conductivity problem," International Journal of Imaging systems and technology, vol. 2, no. 2, pp. 66-75, 1990.

[32] H. Zhao, J. Shi, X. Qi, X. Wang, and J. Jia, "Pyramid scene parsing network," in Proceedings of the IEEE conference on computer vision and pattern recognition, 2017, pp. 2881-2890.

[33] A. G. Howard, M. Zhu, B. Chen, D. Kalenichenko, W. Wang, T. Weyand, M. Andreetto, and H. Adam, "Mobilenets: Efficient convolutional neural networks for mobile vision applications," arXiv preprint arXiv:1704.04861, 2017.

[34] A. Paszke, S. Gross, F. Massa, A. Lerer, J. Bradbury, G. Chanan, T. Killeen, Z. Lin, N. Gimelshein, and L. Antiga, "Pytorch: An imperative style, high-performance deep learning library," Advances in neural information processing systems, vol. 32, pp. 8026-8037, 2019.

[35] Y. Chen and H. Liu, "Location-dependent performance of large-area piezoresistive tactile sensors based on electrical impedance tomography," IEEE Sensors Journal, vol. 21, no. 19, pp. 21 622-21 630, 2021. 